\title{
Identification and Characterization of The Pyruvate Dehydrogenase E1 Gene in The Oriental River Prawn, Macrobrachium Nipponense
}

Shubo Jin

Chinese Academy of Fishery Sciences

Yuning $\mathrm{Hu}$

Nanjing Agricultural University

Hongtuo Fu ( $\nabla$ fuht@ffrc.cn )

Chinese Academy of Fishery Sciences

Sufei Jiang

Chinese Academy of Fishery Sciences

Yiwei Xiong

Chinese Academy of Fishery Sciences

Hui Qiao

Chinese Academy of Fishery Sciences

Wenyi Zhang

Chinese Academy of Fishery Sciences

Yongsheng Gong

Chinese Academy of Fishery Sciences

Yan Wu

Chinese Academy of Fishery Sciences

\section{Research Article}

Keywords: Macrobrachium nipponense, PDHE1, qPCR analysis, RNAi, male sexual development

Posted Date: January 6th, 2021

DOI: https://doi.org/10.21203/rs.3.rs-134507/v1

License: (c) (i) This work is licensed under a Creative Commons Attribution 4.0 International License.

Read Full License 
1 Identification and Characterization of the Pyruvate dehydrogenase E1 Gene in the Oriental

2 River Prawn, Macrobrachium nipponense

3 Shubo Jin ${ }^{1}$, Yuning $\mathrm{Hu}^{2}$, Hongtuo $\mathrm{Fu}^{1,2 *}$, Sufei Jiang ${ }^{1}$, Yiwei Xiong ${ }^{1}$, Hui Qiao ${ }^{1}$, Wenyi

$4 \quad$ Zhang $^{1}$, Yongsheng Gong ${ }^{1}$, Yan $\mathrm{Wu}^{1}$

1. Key Laboratory of Freshwater Fisheries and Germplasm Resources Utilization,

6 Ministry of Agriculture, Freshwater Fisheries Research Center, Chinese Academy of Fishery

$7 \quad$ Sciences, Wuxi 214081, China

8

9

2. Wuxi Fisheries College, Nanjing Agricultural University, Wuxi 214081, China

*Corresponding author: Hongtuo $\mathrm{Fu}$

Freshwater Fisheries Research Center, Chinese Academy of Fishery Sciences

9 Shanshui East Road, Wuxi 214081, Jiangsu Province, P. R. China

E-mail address: fuht@ffrc.cn

Tel.: +86.51085558835

\section{Abstract}

Pyruvate dehydrogenase E1 (PDHE1) was predicted to be involved in the male sexual development in M. nipponense in the previous study. In this study, the functions of Mn-PDHE1 were further analysed in depth using quantitative polymerase chain reaction (qPCR), in situ hybridization, western-blot and RNA interference (RNAi), combined with the histological observations. The full-genome sequence of Mn- PDHE1 was 65,630 base pairs (bp) on Chromosome 11, including 15 introns and 14 exons. The full cDNA sequence of Mn-PDHE1 was 1614 bp, including a 1077 bp open reading frame, encoding for 358 amino acids. qPCR analysis revealed that Mn- PDHE1 showed the highest expression level in the testis, and male 
prawns at post-larval developmental stage 25 (PL25 $\hat{\text { o }}$ ) was higher than PL25우, indicating that

PDHE1 was potentially involved in the male sexual development in $M$. nipponense. An additional function of Mn-PDHE1 was predicted to be involved in the metamorphosis process. In situ hybridization and western-blot indicated that PDHE1 was involved in the whole testis development. The Mn-IAG mRNA was decreased with the decrease of Mn- PDHE1. This indicated PDHE1 has the positive regulatory effects on IAG in M. nipponese. The testis and androgenic gland development were delayed after the injection of Mn- PDHE1 dsRNA, compared with those of control group, indicating PDHE1 has positive effects on the male sexual development in $M$. nipponense. This study highlights the functions of PDHE1 in $M$. nipponense, which can be applied to future studies of the male sexual development in other crustacean species.

Key words: Macrobrachium nipponense, PDHE1, qPCR analysis, RNAi, male sexual development.

\section{Introduction}

The oriental river prawn, Macrobrachium nipponense (Crustacea; Decapoda; Palaemonidae), is widely distributed in China and other Asian countries [1-3]. It is an important commercial species in China with the annual aquaculture production reached of 205,010 tons in 2016 [4]. The male prawn showed better growth performance than their female counterparts. Male prawns grow faster and reach larger size at the harvest time [2]. In addition, the rapid development of testis in the reproductive season is another main problem, restricted the sustainable development of $M$. nipponense. According to the previous studies, the testis of a new born $M$. nipponense can reach sexual maturity within 40 days after hatching [5]. Thus, inbreeding will be happened between the new born prawns, leading to the decrease of the ability of resistance to adversity in their offspring, the small scale of market prawn, and the 
degradation of germplasm resources. Therefore, it is urgently needed to fully understand the male sexual differentiation and development mechanism, with the aims of establishment of the technique to produce all male progeny on a commercial scale, and to regulate the process of testis development in M. nipponense.

Androgenic gland is a special tissue in crustacean species, which plays essential roles in male sexual differentiation and development in crustacean species. The androgenic gland and its secreted hormones have been proven to promote the driving of male sexual differentiation, the establishment of male sexual characteristics, and the development of the testes in crustacean species [6-7]. The previous studies also conformed the multiple and important functions of testis in male sexual differentiation, sexual maturity, and reproductive capability in $M$. nipponense [8-11]. The histological observation of gonad development during the post-larval development stages of $M$. nipponense indicated that the development of androgenic gland had important regulatory roles in testis development in $M$. nipponense [5].

It is widely acknowledged that many environmental factors can regulate the sexual differentiation and the developmental process through affecting the expression profiles of sexrelated genes, especially temperature, illumination, and the presence of chemical pollutants [12]. The histological observations have been proven the significant histological difference in testis and androgenic gland between reproductive season and non-reproductive season [13]. According to the transcriptomic profiling analysis of the testis and androgenic gland between the reproductive season and non-reproductive season in $M$. nipponense, Glycolysis/Gluconeogenesis and TCA were the two main enriched metabolic pathways of differentially expressed genes (DEGs) in both of transcriptomic profiling analysis of the testis and androgenic gland, predicting these two metabolic pathways promote the male sexual differentiation and development in $M$. nipponense through providing ATP. Pyruvate dehydrogenase E1 (PDHE1) is an important DEG, enriched in both of the 
Glycolysis/Gluconeogenesis and tricarboxylic acid cycle (TCA) metabolic pathways [13]. The pyruvate dehydrogenase complex (PDHc) plays essential roles in the glycolysis pathway. Glucose reduced to form pyruvate through glycolysis, and PDHc can catalyse the oxidative decarboxylation of pyruvate to be acetyl-CoA required by the TCA cycle, depending on the needs of cells [14-15]. The deficiency of pyruvate dehydrogenase causes that pyruvate cannot be converted into acetyl-CoA, but reduced to lactic acid, thus affecting the TCA cycle, resulting in the decrease of ATP production [16]. The abnormal of TCA cycle leads to the metabolic disorders and tissue damage. Therefore, PDHc plays an important role in maintaining the normal metabolism of animals. PDHc has three different component enzymes, which were E1, E2 and E3. Pyruvate dehydrogenase E1 (PDHE1) is the key enzyme component of PDHc, which is located at the first one of three PDHc components and catalyses the rate-limiting step of the oxidative decarboxylation of the pyruvate [17-18].

In this study, we aimed to verify the important roles of PDHE1 in the mechanism of male sexual development in M. nipponense, by using RACE cloning, PCR analysis, western-blot, in situ hybridization analysis and RNAi, combined with the histological observations. This study analysed the male sexual development of $M$. nipponense from the energy metabolism for the first time, providing new insight for the analysis of male sexual differentiation and development in M. nipponense, as well as that in the whole crustacean species.

\section{Results}

\section{Genome and cDNA sequence analysis}

The full cDNA sequence of Mn-PDHE1 was 1614 bp, including a 1077 bp open reading frame, encoding for 358 amino acids. The 5' untranslated region (UTRs) and the 3' UTR were $264 \mathrm{bp}$ and $273 \mathrm{bp}$, respectively (Figure 1). The sequence has been submitted to NCBI with the accession number of MW366892. The molecular weight and theoretical isoelectric point of the 
protein were $38.539 \mathrm{kDa}$ and 6.56, respectively. The full genome sequence of Mn-PDHE1 was 65,630 bp on Chromosome 11 at position 59,249,382-59,314742. The full genome sequence included 15 introns and 14 exons.

The BLASTP similarity analysis in NCBI showed that Mn-PDHE1 showed the highest sequence similarity with that of Penaeus vannamei, reached to $90.72 \%$. The similarities with other PDHE1 sequences from other insect species were also over $70 \%$, including Blattella germanica (81.66\%), Zootermopsis nevadensis (81.47\%\%), Brunneria borealis $(77.31 \%)$, and Nicoletia phytophila (79.30\%) (Figure 2). The phylogenetic tree was constructed by using MEGA 5.1, in order to analyse the evolutionary relationship between the amino acid sequences of Mn-PDHE1 and other well-defined PDHE1 sequences in NCBI, followed the maximumlikelihood method. The amino acid sequences listed in Table 1 were used for the construction of phylogenetic tree. The phylogenetic tree was mainly clustered into two groups. The amino acids sequence of Mn-PDHE1 clustered as a group with that of Penaeus vannamei, whereas the amino acid sequences of PDHE1 from insect species were clustered as another group. Thus, Mn-PDHE1 has the closest evolutionary relationship with that of Penaeus vannamei, and dramatically long evolutionary relationship with that from insect species (Figure 3).

\section{Expression analysis in different tissues and developmental stages}

The tissue distributions were evaluated by qPCR. It may reflect the physiological function of a gene. The highest expression of Mn-PDHE1 was observed in testis, followed by the heart and ovary, which showed significant difference with other tested tissues $(\mathrm{P}<0.05)$. The lowest expression was observed in muscle, and the expressions in testis and ovary were 20.37-folder and 13.18-folder higher than that in muscle, respectively (Figure 4-A).

qPCR was also used to evaluated the mRNA expression of Mn-PDHE1 in different larval and post-larval developmental stages. The new born prawns can be can distinguish between 
male and female for the first time at the post-larval developmental stage 25 (PL25). Overall, the expression levels of Mn-PDHE1 were higher in the larval developmental stages, comparted with that of post-larval developmental stages. During the whole larval and post-larval developmental stages, the highest expression level was observed in larval developmental stage 15 (L15), while the lowest expression level of Mn-PDHE1 was observed at female of PL25 (PL25 9 ). The expressions at L15 was 2.87-folder higher than that of PL25 9 . During the postlarval developmental stages, the highest expression level was observed in PL5, and was then gradually decreased to PL15. The expression level in male of PL25 (PL25 $ڤ$ ) was 1.64-folder higher than that of PL25 9 , and showed significant difference $(\mathrm{P}<0.05)$ (Figure 4-B).

\section{Expression analysis in different ovarian developmental stages}

The expression pattern of Mn-PDHE1 was analysed in different ovarian developmental cycle of $M$. nipponense. The expression of Mn-PDHE1 reached the bottom at $\mathrm{O}$ III during the ovarian developmental cycle, and showed significant difference with other tested stages $(\mathrm{P}<0.05)$. The highest expression level was observed in $\mathrm{O}$ V, followed by O I, which is 2.13-folder and 1.98folder higher than that of O III (Figure 5).

\section{Western-blot analysis}

Western-blot analysis was performed in the testis and androgenic gland of reproductive season and non-reproductive season, and in the different reproductive cycle of ovarian development. The western-blot analysis showed that the molecular mass of Mn-PDHE1 was approximately $50 \mathrm{kDa}$, which was slightly larger than that of the predicted molecular weight. It has been proven that the mRNA expressions of Mn-PDHE1 in the testis of the reproductive season were higher than that of the non-reproductive season [13]. As shown in Figure 5, clear bands were observed in the testis of the testis from the reproductive season, while the band in the testis of the non-reproductive season was blurry, indicating the expression of Mn-PDHE1 protein was 
up-regulated in the testis of the reproductive season than that of non-reproductive season. The same expression pattern was observed in the androgenic gland between the reproductive season and non-reproductive season, which was consistent with that of qPCR verification (Figure 6A).

In the different reproductive cycle of ovarian development, clear bands were only observed in the $\mathrm{O} I$ and $\mathrm{O} V$, indicating Mn-PDHE1 was only transcribed in the $\mathrm{O} I$ and $\mathrm{O} V$. The protein expression of Mn-PDHE1 was up-regulated in the $\mathrm{O} V$ than that in the $\mathrm{O}$ I. However, no band was observed in O II, O III and O IV, indicating Mn-PDHE1 protein did not transcribed in these tissues, which was consistent with the qPCR verification (Figure 6-B).

\section{In situ hybridization analysis}

In situ hybridization analysis was performed in the testis, androgenic gland and different reproductive cycle of ovarian development. According to Hematoxylin and eosin (HE) staining, androgenic gland included androgenic gland cells (I, II, III) and the ejaculatory bulb. The cells in the testis included spermatogonia, spermatocyte, sperms and collecting tissues. Strong mRNA signals Mn-PDEH1 in the androgenic gland were only observed in the ejaculatory bulb, while no signals were found in all stages of androgenic gland cells (Figure 7). Strong mRNA signals of Mn-PDHE1 were observed in all of the cell types (Figure 7).

Oogonia and follicle cells, derived from ovarian epithelial cells, were observed in ovary stage I. The follicular cavity then formed in stage II. Oocyte volume gradually increased in stage III, and yolk granules accumulated in the oocyte (stage IV) during oogenesis and vitellogenesis. According to the figure 7, the mRNA signals of Mn-PDHE1 were observed in all of the cell types from O I to O IV, including nucleus, oogoniums, oocytes, cytoplasmic membrane, yolk granule, follicle cells, and follicle membrane (Figure 7).

\section{RNAi analysis}


The potential functions of Mn-PDHE1 in the male prawns were evaluated by using RNAi analysis. The Mn-PDHE1 dsRNA was injected twice and the RNAi experiment was lasted for 2 weeks. The relative mRNA expressions of Mn-PDEH1 were measured in the testis after the Mn-PDHE1 dsRNA treatment. The Mn-PDHE1 mRNA expression remained at a stable level in control group, and showed no significant difference $(\mathrm{P}<0.05)$. However, the Mn-PDHE1 mRNA expression in the RNAi group gradually decreased from day 1 to day 7 , and reached the bottom at day 7. The Mn-PDHE1 mRNA decreased over $95 \%$ and $85 \%$ at day 7 and day 14 in RNAi group, respectively, compared to those in control group at the same day $(P<0.01)$ (Figure 8-A). The relative mRNA expressions of Mn-PDHE1 were also measured in the androgenic gland from the same prawns after the Mn-PDHE1 dsRNA treatment. The expressions of Mn-IAG were decreased with the decrease of Mn-PDHE1. The Mn-PDHE1 mRNA decreased over $49 \%$ and $31 \%$ at day 7 and day 14 in RNAi group, respectively, compared to those in control group at the same day $(P<0.01)$ (Figure 8-B).

According to the HE staining analysis, the sperms were the dominant cells in the testis from the control group, while only limited number of spermatogonias and spermatocytes were observed. The cell types did not show any significant morphological difference between different days in the control group. In the RNAi group, the number of sperms were decreased with the time of the Mn-PDHE1 dsRNA treatment, and sperms were rarely observed at day 14 after However, the number of spermatogonias and spermatocytes were increased (Figure 9). As the same as the histological observations in the testis, the cell types in the androgenic gland of the control group also show no significant morphological difference between different days, and the cells at stage III were the dominant cell type. However, the number of cells at stage III were significantly decreased with the time of the Mn-PDHE1 dsRNA treatment (Figure 10).

\section{Discussion}


The pyruvate dehydrogenase complex (PDHc) is involved in the glycolysis pathway, catalysing the oxidative decarboxylation of pyruvate to be acetyl-CoA, required by the TCA cycle to generate ATP [14-15]. Pyruvate dehydrogenase E1 (PDHE1) is the key enzyme component of PDHc, promoting ATP generation in Glycolysis/Gluconeogenesis and TCA metabolic pathway. PDHE1 was predicted to play important roles in generating ATP for male sexual development in $M$. nipponense in the previous study [13]. In this study, we aimed to focus on the potential functions of PDHE1 in male sexual development through performing the qPCR analysis, western-blot analysis, in situ hybridization and RNAi analysis in M. nipponense. To the data, the actual functions of PDHE1 did not analyse in any crustacean species.

According to the BLASTP similarity analysis in NCBI, the similarity between Mn-PDHE1 and PDHE1 of $P$. vannamei reached to $90.72 \%$, and also shared over $70 \%$ sequence identity with PDHE1 from other insect species. To the best of our knowledge, PDHE1 sequence was only provided in one crustacean species, which was $P$. vannamei. According to the Neighborjoining analysis, the Mn-PDEH1 clustered with P. vannamei as a group, and the PDHE1 amino acids sequence from other insect species were clustered as another group. The Mn-PDEH1 has the closest evolutionary relationship with that from crustacean species, and long evolutionary relationship with that of insect species, which was consistent with evolutionary analysis of other genes in M. nipponense [19-21]. In order to better analyse the evolutionary relationship of Mn-PDHE1, more PDHE1 sequences from other species were suggested to be provided, especially for that in the crustacean species.

The physiological functions of PDHE1 in M. nipponense can be reflected by the qPCR analysis in various adult tissues and developmental stages. To the best of our knowledge, the qPCR analysis of PDHE1 in other species were rarely. QPCR analysis of PDHE1 in Ascaris suum revealed that Type I sequence was highly expressed in adult muscle, and Type II sequence was abundant in third-stage larvae as well as in adult muscle [22]. In Streptococcus mutans, 
PDHE1 $\alpha$ expression dramatically increased in the condition of acidic growth and acid adaptation. Additionally, PDHE1 $\alpha$ expression increased in the conditions of favouring heterofermentative growth; decreased in the presence of excess glucose, and increased during stationary phase compared with mid-log phase growth, indicating that PDHE1 $\alpha$ expression of S. mutans favourite in the condition of heterofermentation and decreased in the presence of glucose [23]. In adult mouse, PDHE1 $\alpha$ in testis showed the expression pattern of the testisspecific and somatic forms [24]. The expression of PDHE1 $\alpha$ was detected in spermatogonia, Leydig cells and Sertoli cells at a low level in somatic form. In situ hybridization and immunostaining analysis showed that PDHE1 $\alpha$ was abundant in spermatocytes. In this study, the highest expression of Mn-PDHE1 was observed in the testis, indicating the activities of

TCA cycle was sufficient to generate ATP, promoting the male sexual development in $M$. nipponense. In different developmental stages of $M$. nipponense, Mn-PDHE1 showed higher expression level in the larval developmental stages than that of the post-larval developmental stages, and the highest expression was observed in L15, indicating PDHE1 promote the metamorphosis process in M. nipponense [25-26]. The highest expression level of Mn-PDHE1 was observed in PL5 during the post-larval developmental stages, and gradually decreased to PL 15. Histological observations have been proven that both of testis and ovary were differentiated from PL5, and matured at 19 and 22, respectively [5]. The highest expression in PL5 indicated that PDHE1 played essential roles in promoting the gonad differentiation. The gender of M. nipponense can be distinguished for the first time by naked eye at PL25, and the expression in PL25 ${ }^{\lambda}$ was 2-folder higher than that in PL25우, which was consistent with that qPCR analysis in different tissues, playing more essential roles in the male sexual development in M. nipponense.

The western-blot analysis of PDHE1 did not performed in any species. Western-blot revealed that the molecular weight of Mn-PDHE1 protein was at about $50 \mathrm{kDa}$, which was 
slightly larger than that of predicted molecular weight, indicating the Mn-PDHE1 protein was modified after transcription. Our previous study has been proven that Mn-PDHE1 mRNA was higher expressed in the testis and androgenic gland from reproductive season than that of nonreproductive season [13]. Western-blot analysis indicated that the protein expression of MnPDHE1 was up-regulated in the testis and androgenic of reproductive season, compared with those of non-reproductive season, which was consistent with the qPCR verification, indicating PDHE1 promotes the testis development in reproductive season. Mn-PDHE1 showed the highest expression level in the $\mathrm{O} \mathrm{V}$, followed by the $\mathrm{O}$ I during the reproductive cycle of ovarian development, while the lowest expression was observed in O III, indicating PDHE1 played more essential roles in activating the ovarian development [27]. Western-blot analysis in different reproductive cycle of ovarian development indicated that the Mn-PDHE1 protein was only transcribed in the $\mathrm{O} I$ and $\mathrm{O} \mathrm{V}$, and $\mathrm{O} \mathrm{V}$ was up-regulated than that of $\mathrm{O}$, which was consistent with the qPCR analysis. Oogonia and follicle cells were observed in ovary stage I, which were derived from ovarian epithelial cells. In $\mathrm{O} V$, the matured oocytes were excreted $[28]$. observed in all of the cell types in the testis, indicating PDHE1 plays essential roles in the by the androgenic gland cells in $M$. nipponense, but it plays essential roles in maintaining the normal structure and function of androgenic gland in M. nipponense [19-21]. In ovary, PDHE1 was widely observed in all cell types, indicating indicating PDHE1 was involved in the whole ovarian developmental process [28]. 

RNA molecules in the cell's cytoplasm [29-31]. RNAi has been widely used in gene function analysis in $M$. nipponense [28, 32]. PDHE1 was involved in the metabolic pathways of Glycolysis/Gluconeogenesis and tricarboxylic acid cycle (TCA), promoting the ATP generation, which has been proven to play essential roles in the testis development in adult mouse. The previous study was predicted that PDHE1 was predicted to be involved in the male sexual development in M. nipponense [13]. RNAi was used to analyse the potential functions of PDHE1 in the mechanism of male sexual development in M. nipponense. qPCR analysis revealed that the mRNA expressions of Mn-PDHE1 were significantly decreased at day 7 and day 14 after the treatment of Mn- PDHE1 dsRNA, indicating the Mn-PDHE1 dsRNA in this study was efficient to knockdown the Mn- PDHE1 expression. In order to analyse the regulatory roles of PDHE1 on IAG, the expression levels of Mn-IAG were also measured in the androgenic gland from the same prawn, treated by Mn- PDHE1 dsRNA. The expressions of Mn-IAG were decreased with the decrease of Mn- PDHE1, indicating PDHE1 has positive regulatory roles on IAG in $M$. nipponense. The IAG gene is a hormone, secreted by the androgenic gland. Previous studies indicated that it plays essential roles in the male sexual differentiation and development in many crustacean species [33-35]. Knockdown the expression of IAG resulted in the sex reverse in $M$. rosenbergii [36]. The positive regulatory relationship between PDHE1 and IAG also indicated the important functions of PDHE1 in the male sexual development in $M$. nipponense, which was as the same as the previous study [13]. The morphological differences of testis and androgenic gland between RNAi group and control group were measured by histological observations. The histological observations in the testis indicated that the number of sperms were decreased with the time of Mn- PDHE1 dsRNA treatment, and sperms were hardly observed at day 14 of Mn- PDHE1 dsRNA treatment, indicating Mn- PDHE1 has positive effects on the testis development in M. nipponense. In 
androgenic gland, the number of cells at stage III were decreased with the time of Mn- PDHE1 dsRNA treatment, indicating Mn- PDHE1 can also promote androgenic gland development in M. nipponense.

In conclusion, the important functions of PDHE1 were further analysed in M. nipponense in this study. The overall results indicated that PDHE1 was predicted to be involved in the metamorphosis, and gonad development in $M$. nipponense, especially that for the male sexual development. Mn- PDHE1 was proven to have positive regulatory effects on the testis and androgenic gland development by RNAi analysis, combined with the histological observations. This study analyses the male sexual development from energy metabolism for the first time in M. nipponense, promoting the future studies of male sexual development in other crustacean species as well.

\section{Materials and Methods}

\section{Ethics statement}

All experiments involving $M$. nipponense in this study have been approved by Institutional Animal Care and Use Ethics Committee of the Freshwater Fisheries Research Center, Chinese Academy of Fishery Sciences (Wuxi, China).

\section{Sample preparation}

The samples were collected followed by the previous study of our lab. Briefly, different tissues were collected from the healthy adult $M$. nipponense obtained from Tai Lake in Wuxi, China $\left(120^{\circ} 13^{\prime} 44^{\prime \prime E}, 31^{\circ} 28^{\prime} 22^{\prime \prime} \mathrm{N}\right)$, including testis, ovary, hepatopancreas, muscle, eyestalk, gill, heart and brain. Specimens for the different stages of larval and post-larval developmental stages were from the full-sibs population, collected with their maturation process. The various phases of ovarian reproductive cycle were obtained according to the standard of previous reports [37]. The reproductive season of testis and androgenic gland were collected at 28 in 
summer for western-blot analysis, while the nonreproductive season of tests and androgenic

317 gland were collected at 15 in winter. The samples were treated with phosphate buffer saline (PBS), and immediately frozen in liquid nitrogen until used for RNA and protein extraction to prevent total RNA and protein degradation.

\section{Rapid Amplification of cDNA Ends (RACE)}

321

The procedures for RACE cloning have been well-described in previous study $[19,38]$. Briefly, the total RNA was extracted from testis using RNAiso Plus Reagent (Takara Bio Inc.). The 3'Full RACE Core Set Ver.2.0 kit and the 5'-Full RACE kit (Takara Bio Inc., Japan) were used to synthesize the templates for $3^{\prime} \mathrm{cDNA}$ and $5^{\prime} \mathrm{cDNA}$ cloning. The specific primers used for Mn-PDHE1 cloning were designed in Primer-BLAST tool in NCBI (http://www.ncbi.nlm.nih.gov/tools/primer-blast/), and listed in Table 2. The BLASTX and BLASTN search program (http://www.ncbi. nlm.nih.gov/BLAST/) and the ORF Finder tool (http://www.ncbi.nlm.nih.gov/gorf/gorf.html) were employed to analyse the structural characteristics. The phylogenetic tree was constructed by MEGA X, followed by the maximum-likelihood method with Bootstrap method of 1000 replications.

\section{qPCR analysis}

qPCR was used to measure the relative mRNA expression of Mn-PDHE1 in different tissues, different developmental stages, and various reproductive cycle of ovary. The Bio-Rad iCycler iQ5 Real-Time PCR System (Bio-Rad) was used to carry out the SYBR Green RT-qPCR assay. The procedure has been well described in details in previous studies [19, 38]. The primers used for qPCR analysis were listed in Table 2. EIF was used as reference gene in this study [39]. The testis templates include undiluted, two times diluted, four times diluted and eight times diluted sample. The slope of the Mn-PDHE1 and EIF at different concentrations of diluted samples were 1.334 and 1.445 , respectively. Thus, the amplification efficiency between the 
target gene and EIF are the same in this study. The tissue with lowest expression level was set as 1 (a relative criterion), and other tissues were then compared with the relative criterion.

\section{In situ Hybridization}

In situ hybridization was performed to analyse the mRNA locations of Mn-PDHE1 in different tissues, including various reproductive cycle of ovary, the testis and androgenic gland in reproductive season. Primer5 software was used to design the anti-sense and sense probes of CISH (Chromogenic in-situ hybridization) study with DIG signal based on the cDNA sequence of each gene. The sequences of anti-sense and sense probes were listed in Table 2, and synthesized by Shanghai Sangon Biotech Company. The detailed procedures of in situ hybridization have been well described in previous studies $[19,28]$. Slides were examined under light microscope for evaluation.

\section{Western-blot analysis}

Testis, androgenic gland, and different ovarian developmental stages samples (20 mg) were respectively obtained in the reproductive season and non-reproductive season. The Bradford [40] method was used to quantify the total protein concentration. The detailed procedure of western-blot was well described in previous study [41]. Briefly, an equal amount of protein from each sample $(50 \mathrm{mg}$ ) was separated on a 10\% SDS-polyacrylamide (SDS-PAGE) and transferred to a PVDF membrane (Millipore, Bedford, MA, United States).

\section{RNA interference (RNAi) analysis}

RNAi was performed to analysis the potentially regulatory roles on Mn- PDHE1 in male sexual development in M. nipponense. Snap Dragon tools was used to design the specific RNAi primer with T7 promoter site (http://www.flyrnai.org/cgibin/RNAifind_primers.pl), and shown in used to synthesize the Mn- PDHE1 dsRNA, followed by the procedures of the manufacturer. 
A total of 300 health mature male $M$. nipponense were collected with body weight of 3.34-

365

366

367 4.56g, and divided into two groups. As described in previous study [28, 42], the prawns from experimental group were injected with $4 \mu \mathrm{g} / \mathrm{g}$ Mn- PDHE1 dsRNA, while the prawns from control group were injected with equal volume of GFP. The PDHE1 mRNA expression were investigated in testis by qPCR after the injection of 1,7 and 14 days, in order to detect the interference efficiency $(\mathrm{N} \geq 5)$.

\section{Histological observation}

The morphological changes of the testis between different days after RNAi treatment was observed by Hematoxylin and eosin (HE) staining. Five testicular samples were respectively collected after 1, 4, 7, 10 and 14 days of RNAi treatment for HE staining. The procedures have been well described in previous studies [43-44]. Olympus SZX16 microscope was used to observe the slides (Olympus Corporation, Tokyo, Japan). The various cell types were labelled based on morphological analysis [5].

\section{Statistical Analysis}

Quantitative data were expressed as mean \pm SD. Statistical differences were estimated by oneway ANOVA followed by LSD and Duncan's multiple range test. All statistics were measured using SPSS Statistics 13.0. A probability level of 0.05 was used to indicate significance $(P<$ $0.05)$.

Acknowledgements: This research was supported by grants from the National Key R\&D Program of China (2018YFD0900201); Central Public-interest Scientific Institution Basal Research Fund CAFS (2019JBFM02, 2019JBFM04); the National Key R\&D Program of China (2018YFD0901303); Jiangsu Agricultural Industry Technology System (JFRS-02); the National Natural Science Foundation of China (31572617); the China Agriculture Research 
System-48 (CARS-48); the New cultivar breeding Major Project of Jiangsu province

(PZCZ201745).

\section{Reference}

390

391

392

393

1. Cai, Y., \& Shokita, S. Report on a collection of freshwater shrimps (Crustacea: Decapoda: Caridea) from the Philippines, with descriptions of four new species. Raffles B. Zool. 54, 245-270 (2006).

2. Ma, K.Y., Feng, J.B., Lin, J.Y., \& Li, J.L. The complete mitochondrial genome of Macrobrachium nipponense. Gene. 487(2), 160-165 (2011).

3. Salman, S.D., Page, T.J., Naser, M.D., \& Yasser, A.G. The invasion of Macrobrachium nipponense (De Haan, 1849) (Caridea: Palaemonidae) into the southern Iraqi marshes. Aquat. Invasions 1(3), 109-115 (2006).

4. Zhao, X.W. et al. Fisheries economic statistics. In: China Fishery Yearbook. Beijing, China, China Agricultural Press., 236 pp (2016).

5. Jin, S.B. et al. Histological observation of gonadal development during post-larva in oriental river prawn, Macrobrachium nipponense. Chin. J. Fish. 29(4), 11-16 (2016).

6. Sagi, A., Cohen, D., \& Wax, Y. Production of Macrobrachium rosenbetgii in momosex population: yield characteristes under intensive monoculture conditions in cages. Aquaculture. 51(3-4), 265-275 (1986).

7. Sagi, A., Cohen, D., \& Milner, Y. Effect of androgenic gland ablation on morphotypic differentiation and sexual characteristics of male freshwater prawns, Macrobrachium rosenbergii. Gen. Comp. Endocr. 77, 15-22 (1990).

8. Cao, J.X. The molecular mechanism in male reproductive tract of the prawn, Macrobrachium rosenbergii. Zhejiang University, 2006. 
9. Guo, Z.H. Study on proliferation and differentiation of spermatogenic cells from Macrobrachium nipponense in vitro. Hebei University, 2007.

10. Qiu, G.F., Du, N.S., \& Lai, W. Studies on the male reproductive system of the freshwater prawn, Macrobrachium nipponense. J. Shanghai Fish. Univ. 4, 107-111 (1995).

11. Yang, W.X., Du, N.S., \& Lai, W. Functional 646 relationship between spermatogenic cells and Sertoli cells during spermatogenesis of freshwater shrimp, Macrobrachium nipponense. Acta Zool. Sin. 45(2), 178-186 (1999).

12. Wedekind, C. Demographic and genetic consequences of disturbed sex determination. Philos. Trans. R. Soc. Lond. B. Biol. Sci. 372, 1729 (2017).

13. Jin, S.B. et al. Analysis of testis metabolome and transcriptome from the oriental river prawn (Macrobrachium nipponense) in response to different temperatures and illumination times. Comp. Biochem. Phys. D. 34, 100662 (2020).

14. Berg, A., \& De Kok, A. 2-Oxo acid dehydrogenase multienzyme complex. The central role of the lipoyl domain. Baiol. Chem. 378, 617-634 (1997).

15. Harris, R.A., Bowker-kinley, M.M., Huang, B.L., \& Wu, P.F. Regulation of the activity of the pyruvate dehydrogenase complex. Adv Enzyme Regul. 42, 249-259 (2015).

16. Carlsson, J., Kujala, U., \& Edlund, M.J.K. Pyruvate dehydrogenase activity in Streptococcus mutans. Infect Immun. 49, 674-678 (1985).

17. Snoep, J.L. et al. Differences in sensitivity to NADH of purified pyruvate dehydrogenase complexes of Enterococcus faecalis, Lactococcus lactis, Azotobacter vinelandii and Escherichia coli: implications for their activity in vivo. FEMS Microbiol. Lett. 114, 279284 (1993).

18. Modak, J., Wolf-Dieter, D., \& Zeng, A.P. Metabolic control analysis of eurkaryotic pyruvate dehydrogenase multienzyme complex. Biotechnol. Prog. 18, 1157-1169 (2002). 
19. Jin, S.B. et al. Molecular Cloning, Expression, and In Situ Hybridization Analysis of Forkhead Box Protein L2 during Development in Macrobrachium nipponense. J. World Aquacult. Soc. 49(2), 429-440 (2018).

20. Jin, S.B. et al. Potential functions of Gem-associated protein 2-like isoform X1 in the oriental river prawn Macrobrachium nipponense: Cloning, qPCR, in situ hybridization, and RNAi analysis. Int. J. Mol. Sci. 20, 3995 (2019).

21. Jin, S.B. et al. Identification of potentially novel functions of DNA polymerase zeta catalytic subunit in oriental river prawn, Macrobrachium nipoponense: Cloning, qPCR, in situ hybridization and RNAi analysis. 3 Biotech 9, 330 (2019).

22. Johnson, K.R., Komuniecki, R., Sun, Y.H., \& Wheelock, M.J. Characterization of cDNA clones for the alpha subunit of pyruvate dehydrogenase from Ascaris suum. Mol. Biochem. Parasit. 51, 37-48 (1992).

23. Korithoski, B., Levesque, C.M., \& Cvitkovitch, D.G. The involvement of the pyruvate dehydrogenase E1asubunit, in Streptococcus mutans acid tolerance. FEMS Microbiol. Lett. 289, $13-19$ (2008).

24. Takakubo, F., \& Dahl, H.M. The Expression Pattern of the Pyruvate Dehydrogenase El $\alpha$ Subunit Genes during Spermatogenesis in Adult Mouse. Exp. Cell Res. 199, 39-49 (1992).

25. Zhang, Y.P. et al. Molecular cloning and expression analysis of transformer- 2 gene during development in Macrobrachium nipponense (de Haan 1849). J. World Aquacult. Soc. 44(3), 338-349 (2013).

26. Zhang, Y.P. et al. Molecular cloning and expression analysis of extra sex combs gene during development in Macrobrachium nipponense. Turk. J. Fish. Aquat. Sc. 13, 331-340 (2013). 
27. Hu, Y.N. et al. Functional Analysis of a SoxE Gene in the Oriental Freshwater Prawn, Macrobrachium nipponense by Molecular Cloning, Expression Pattern Analysis, and in situ Hybridization (de Haan, 1849). 3 Biotech 10(1): 10 (2020).

28. Li, F. et al. Identification and characterization of opsin gene and its role in ovarian maturation in the oriental river prawn Macrobrachium nipponense. Comp. Biochem. Physiol. B. 218, 1-12 (2018).

29. Jones, H.D. Wheat transformation: current technology and applications to grain development and composition. J. Cereal Sci., 41(2), 137-147 (2005).

30. Kusaba, M. RNA interference in crop plants. Curr. Opin. Biol. 15, 139-143 (2004).

31. Smith, C.A. et al. The avian Z-linked gene DMRT1 is required for male sex determination in the chicken. Nature 461, 267-271 (2009).

32. Li, F.J. et al. Molecular characterization of insulin-like androgenic gland hormone-binding protein gene from the oriental river prawn Macrobrachium nipponense and investigation of its transcriptional relationship with the insulin-like androgenic gland hormone gene. Gen. Comp. Endocr. 216(6), 152-160 (2015).

33. Rosen, O. et al. A sexual shift induced by silencing of a single insulin-like gene in crayfish: ovarian upregulation and testicular degeneration. PLoS One 5, e15281 (2010).

34. Ventura, T. et al. Temporal silencing of an androgenic gland-specific insulin-like gene affecting phenotypical gender differences and spermatogenesis. Endocrinology 150, 12781286 (2009).

35. Ventura, T. et al. Expression of an androgenic gland-specific insulin-like peptide during the course of prawn sexual and morphotypic differentiation. ISRN Endocrinol. 2011, 476283 (2011). 
36. Ventura, T. et al. Timing sexual differentiation: full functional sex reversal achieved through silencing of a single insulin-like gene in the prawn, Macrobrachium rosenbergii. Biol. Reprod. 86, 90 (2012).

37. Qiao, H. et al. Characterization, expression, and function analysis of gonad-inhibiting hormone in Oriental River prawn, Macrobrachium nipponense and its induced expression by temperature. Comp. Biochem. Phys. A. 185, 1-8 (2015).

38. Jin, S.B. et al. Molecular cloning of two tropomyosin family genes and expression analysis during development in oriental river prawn, Macrobrachium nipponense. Gene. 546, 390-397 (2014).

39. Hu, Y.N. et al. Validation and evaluation of reference genes for Quantitative real-time PCR in Macrobrachium nipponense. Int. J. Mol. Sci. 19(8), 2258 (2018).

40. Bradford, M.M. A rapid and sensitive method for the quantification of microgram quantities of protein utilizing the principle of protein-dye binding. Anal. Biochem. 72, 248-254 (1976).

41. Sun, S.M. et al. Molecular cloning, mRNA expression and characterization of membrane bound hemoglobin in oriental river prawn Macrobrachium nipponense. Comp. Biochem. Physiol. A Mol. Integr. Physiol. 207, 36-42 (2017).

42. Jiang, F.W. et al. The RNA Interference Regularity of Transformer-2 Gene of Oriental River Prawn Macrobrachium nipponense. Chinese Agricultural Science Bulletin 30(32), 32-37 (2014).

43. Ma, X.K., Liu, X.Z., Wen, H.S., Xu, Y.J., \& Zhang, L.J. Histological observation on gonadal sex differentiation in Cynoglossus semilaevis Günther. Mar. Fish. Res. 27(2), 5561 (2006).

44. ShangGuan, B.M., Liu, Z.Z., \& Li, S.Q. Histological Studies on Ovarian Development in Scylla serrata. J. Fish. China. 15(2), 96-103 (1991). 
Fig. 1: Nucleotide and deduced amino acid sequence of Mn-PDHE1. The nucleotide sequence is displayed in the $5^{\prime}-3^{\prime}$ directions and numbered at the left. The deduced amino acid sequence is shown in a single capital letter amino acid code. $3^{\prime}$ UTR and $5^{\prime}$ UTR are shown with lowercase letters. Codons are numbered at the left with the methionine (ATG) initiation codon, an asterisk denotes the termination codon (TGA).

Fig. 2: The alignment of amino acid sequences of PDHE1 from different species.

Figure 3: The phylogenetic tree of PDHE1 from different organisms based on amino acid sequence comparisons. Species names and types of PDHE1 are listed on the right of the tree. Red rectangles indicated $M$. nipponense.

Fig. 4-A: Expression characterization of $M n$ - PDHE1 in different tissues. Fig. 4-B: Expression characterization of $M n$ - $P D H E 1$ in different developmental stages. The amount of $M n$ - PDHE1 mRNA was normalized to the EIF transcript level. Data are shown as mean +SD (standard deviation) of tissues from three separate individuals. Capital letters indicate expression difference between different samples.

Fig. 5: Expression characterization of $M n-P D H E 1$ in different reproductive cycles of ovary. The amount of $M n$ - PDHE1 mRNA was normalized to the EIF transcript level. Data are shown as mean $+\mathrm{SD}$ (standard deviation) of tissues from three separate individuals. Capital letters indicate expression difference between different samples.

Fig. 6-A: Western-blot analysis of Mn-PDHE1 in the testis and androgenic gland from the reproductive season and non-reproductive season. Line 1: Testis from the non-reproductive season; Line 2: Testis from the reproductive season; Line 3: Androgenic gland from reproductive season; Line 4: Androgenic gland from the non-reproductive season. Fig. 6-B: Western-blot analysis of Mn-PDHE1 in the different ovarian developmental stages. Line 1: Ovarian developmental stage I; Line 2: Ovarian developmental stage II; Line 3: Ovarian 
developmental stage III; Line 4: Ovarian developmental stage IV; Line 5: Ovarian developmental stage V. B-action was used as reference gene.

531 Fig. 7: Location of PDHE1 gene was detected in testis, androgenic gland and ovary of $M$. 532 nipponense by using in situ hybridization. ST: seminiferous tubule; SG: Spermatogonia; SC: spermatocyte; S: sperm; CT: collecting tissue; E: wall epithelium; EM: eosinophilic matrix; VD: vas deferens; EB: ejaculatory bulb; OG: oogonium; OC: oocyte; CM: cytoplasmic membrane; N: nucleus; Y: yolk granule; FC: follicle membrane. Scale bars $=20 \mu \mathrm{m}$.

Fig. 8-A: Expression characterization of $M n$ - $P D H E 1$ at different days after $M n$ - PDHE1 dsRNA injection. Fig. 8-B: Expression characterization of $M n-I A G$ at different days after $M n$ PDHE1 dsRNA injection. The amount of $M n-P D H E 1$ and $M n-I A G$ mRNA was normalized to the EIF transcript level. Data are shown as mean +SD (standard deviation) of tissues from three separate individuals. Capital letters indicated expression difference between different days after vehicle injection in control group. Lowercase indicated expression difference between different days after $M n-P D H E 1$ dsRNA injection in RNAi group. * $(\mathrm{P}<0.05)$ and ** $(\mathrm{P}<0.01)$ indicates significant expression difference between the RNAi group and control group at the sample day.

Fig. 9: The histological observations of testis between RNAi and control group. SG: Spermatogonia; SC: spermatocyte; S: sperm; CT: collecting tissue. Scale bars $=20 \mu \mathrm{m}$.

Fig. 10: The histological observations of testis between RNAi and control group. EB: ejaculatory bulb; I: Cell stage I of androgenic gland; II: Cell stage II of androgenic gland; III:

549 Cell stage III of androgenic gland. Scale bars $=20 \mu \mathrm{m}$. 
553 Table 1. Universal and specific primers used in this study

\begin{tabular}{|c|c|c|}
\hline Primer name & Nucleotide Sequence $\left(5^{\prime} \rightarrow 3^{\prime}\right)$ & Purpose \\
\hline PDE1-3GSP1 & CTGAGATCTGTGCTAGAATAG & FWD first primer for GEM $3^{\prime}$ RACE \\
\hline PDE1-3GSP2 & CCCAGTGATTCGTGTAACGGG & FWD second primer for $G E M 3^{\prime}$ RACE \\
\hline PDE1 -5GSP1 & TCAGGAACACCCCTTCATCCC & RVS first primer for $G E M 5^{\prime} \mathrm{RACE}$ \\
\hline PDE1 -5GSP2 & TACTCTTGTTGTGGAGAAACT & RVS second primer for $G E M 5^{\prime}$ RACE \\
\hline 3'RACE OUT & TACCGTCGTTCCACTAGTGATTT & RVS first primer for $3^{\prime}$ RACE \\
\hline 3'RACE IN & CGCGGATCCTCCACTAGTGATTTCACTATAGG & RVS second primer for $3^{\prime}$ RACE \\
\hline 5'RACE OUT & CATGGCTACATGCTGACAGCCTA & FWD first primer for $5^{\prime}$ RACE \\
\hline 5'RACE IN & CGCGGATCCACAGCCTACTGATGATCAGTCGATG & FWD second primer for $5^{\prime}$ RACE \\
\hline SDHB -RTF & TGACCTTAACGGCAACGAGG & FWD primer for $G E M$ expression \\
\hline SDHB -RTR & TCCAGGGCAGAATTGAGAGC & RVS primer for $G E M$ expression \\
\hline EIF-F & CATGGATGTACCTGTGGTGAAAC & FWD primer for $\beta$-actin expression \\
\hline EIF-R & CTGTCAGCAGAAGGTCCTCATTA & RVS primer for $\beta$-actin expression \\
\hline PDE1 anti-sense Probe & GTTGACCTGCTGCTACTCTTGTTGTGGAGAAACTACGG & Probe for GEM ISH analysis \\
\hline PDE1 sense Probe & CCGTAGTTTCTCCACAACAAGAGTAGCAGCAGGTCAAC & Probe for GEM ISH analysis \\
\hline PDE1 RNAi-F & TAATACGACTCACTATAGGGGTGCTCTTAGCACTGGAGGC & FWD primer for RNAi analysis \\
\hline PDE1 RNAi-R & TAATACGACTCACTATAGGGCCAAGTAGTGGAAGGCAGGA & RVS primer for RNAi analysis \\
\hline
\end{tabular}

554

555

556

557

558 


\begin{tabular}{ll}
\hline Species & Accession number \\
\hline Macrobrachium nipponense & MW366892 \\
Penaeus vannamei & ROT67345.1 \\
Blattella germanica & PSN33167.1 \\
Zootermopsis nevadensis & XP_021917776.1 \\
Brunneria borealis & QBH74078.1 \\
Orthoderella ornate & QBH74091.1 \\
Nicoletia phytophila & QBH74090.1 \\
Thermobia domestica & QBH74092.1 \\
Atta cephalotes & XP_012055795.1 \\
Atta colombica & XP_018049360.1 \\
Bemisia tabaci & XP_018907986.1 \\
Aedes albopictus & Aedes albopictus XP
\end{tabular}

561

562 


\section{Figures}

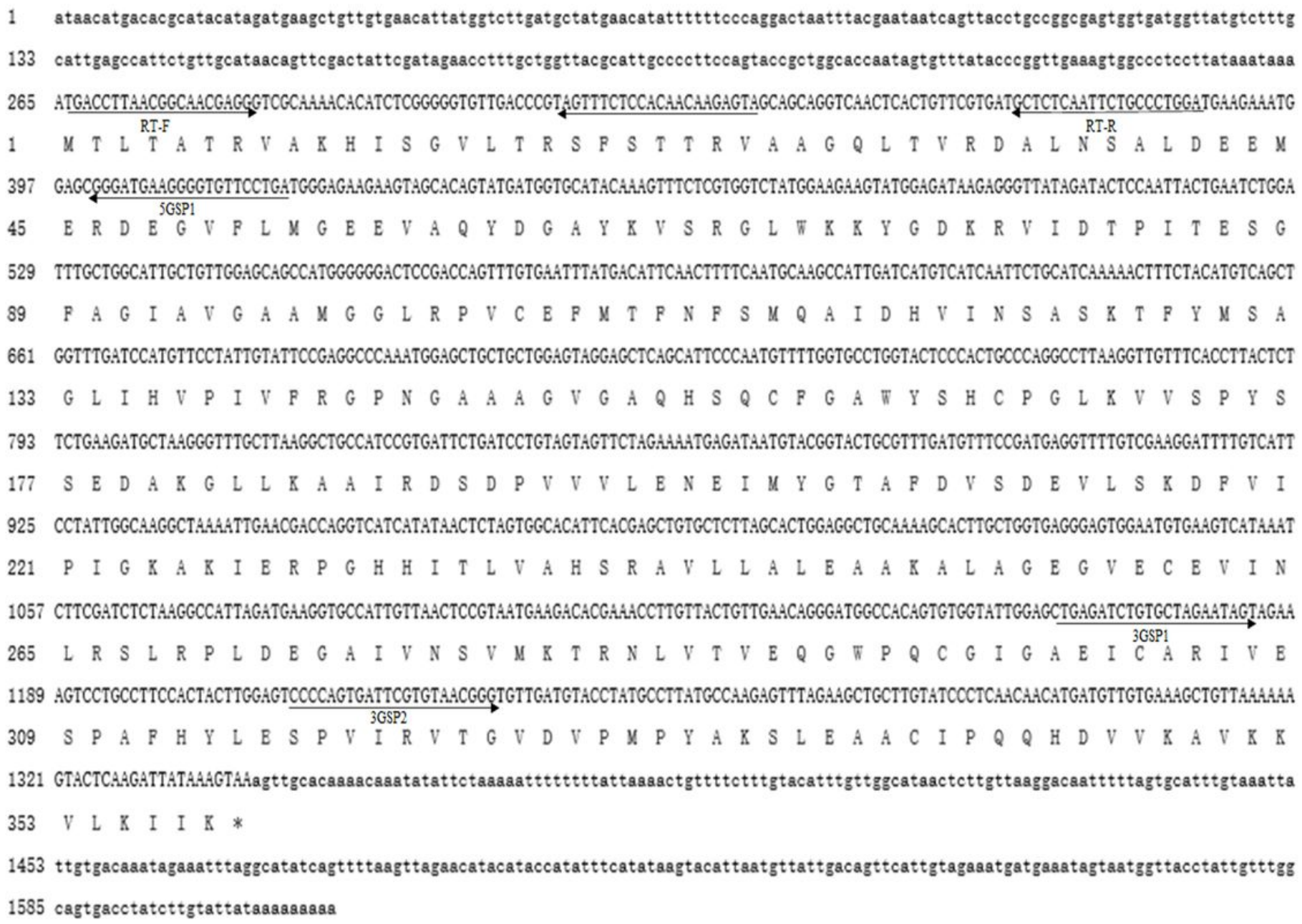

\section{Figure 1}

Nucleotide and deduced amino acid sequence of Mn-PDHE1. The nucleotide sequence is displayed in the $5^{\prime}-3^{\prime}$ directions and numbered at the left. The deduced amino acid sequence is shown in a single capital letter amino acid code. 3' UTR and 5' UTR are shown with lowercase letters. Codons are numbered at the left with the methionine (ATG) initiation codon, an asterisk denotes the termination codon (TGA). 
Macrobrachium nipponense Penaeus vannamej Blattelìa_germanica Zootermopsis nevadensis Brunneria borealis Orthodereilla_ornate Nicoletia_phytophile Thermobia domestica Atta cephālotes Atta_colombica Bemisia tabaci Aedes albopictus Consensus

Macrobrachium nipponense Penaeus_vannamei Blattella germanica zootermopsis nevadensis Brunneria_borealis Orthoderella ornate Nicoletia phytophile Thermobia_domestica Atta_cephalotes

Atta colombica

Bemisia tabaci Aedes_albopictus Consensus

Macrobrachium nipponense Penaeus vannamei

Blattelīa_germanica

zootermopsis_nevadensis

Brunneria borealis

Orthoderella ornate

Nicoletia_phytophile Thermobia domestica

Atta cephalotes

Atta_colombica

Bemisia tabaci

Aedes albopictus

Conseñsus

Macrobrachium nipponense Penaeus vannamei

Blattelīa_germanica

Zootermopsis nevadensis

Brunneria borealis

Orthoderella_ornate

Nicoletia phytophile

Thermobia domestica

Atta_cephälotes

Atta colombica

Bemisia tabaci

Aedes_albopictus

Consensus

Macrobrachium nipponense Penaeus vannamei

Blattella germanica

Zootermopsis_nevadensis

Brunneria borealis

Orthoderella ornate

Nicoletia_phȳtophile

Thermobia_domestica

Atta cephalotes

Atta colombica

Bemisia_tabaci

Aedes albopictus

Consensus
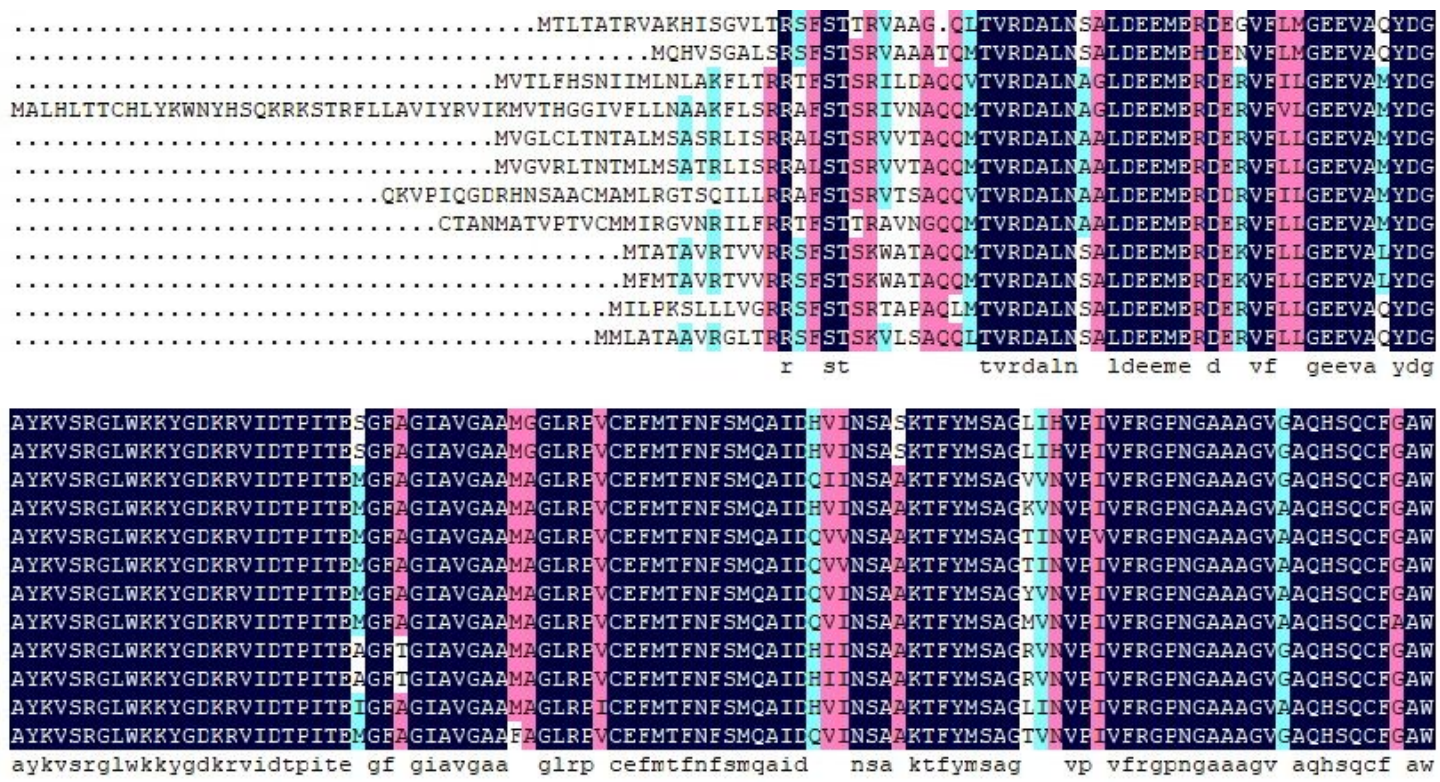

162

155

166

200

166

166

174

170

157

157

158

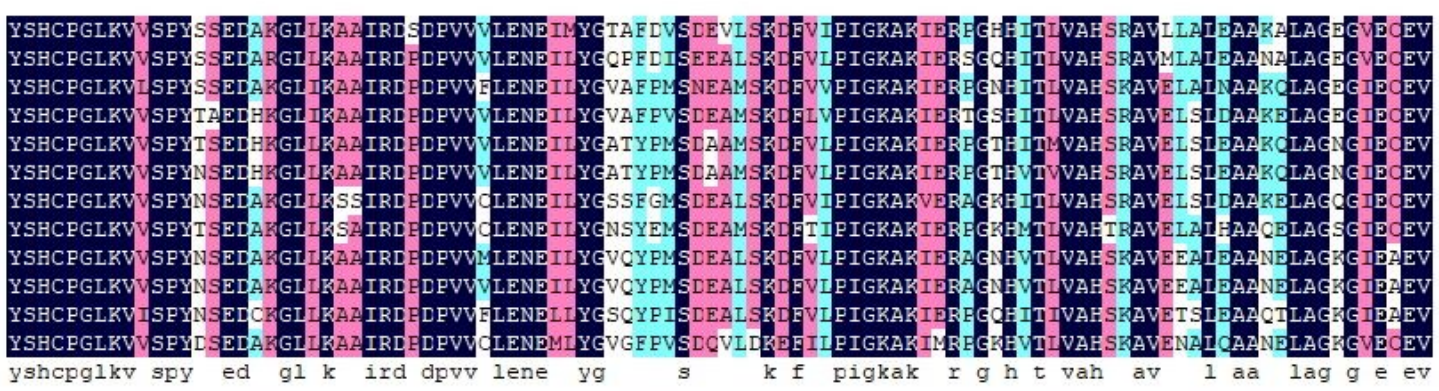

262

255

266

300

266

266

274
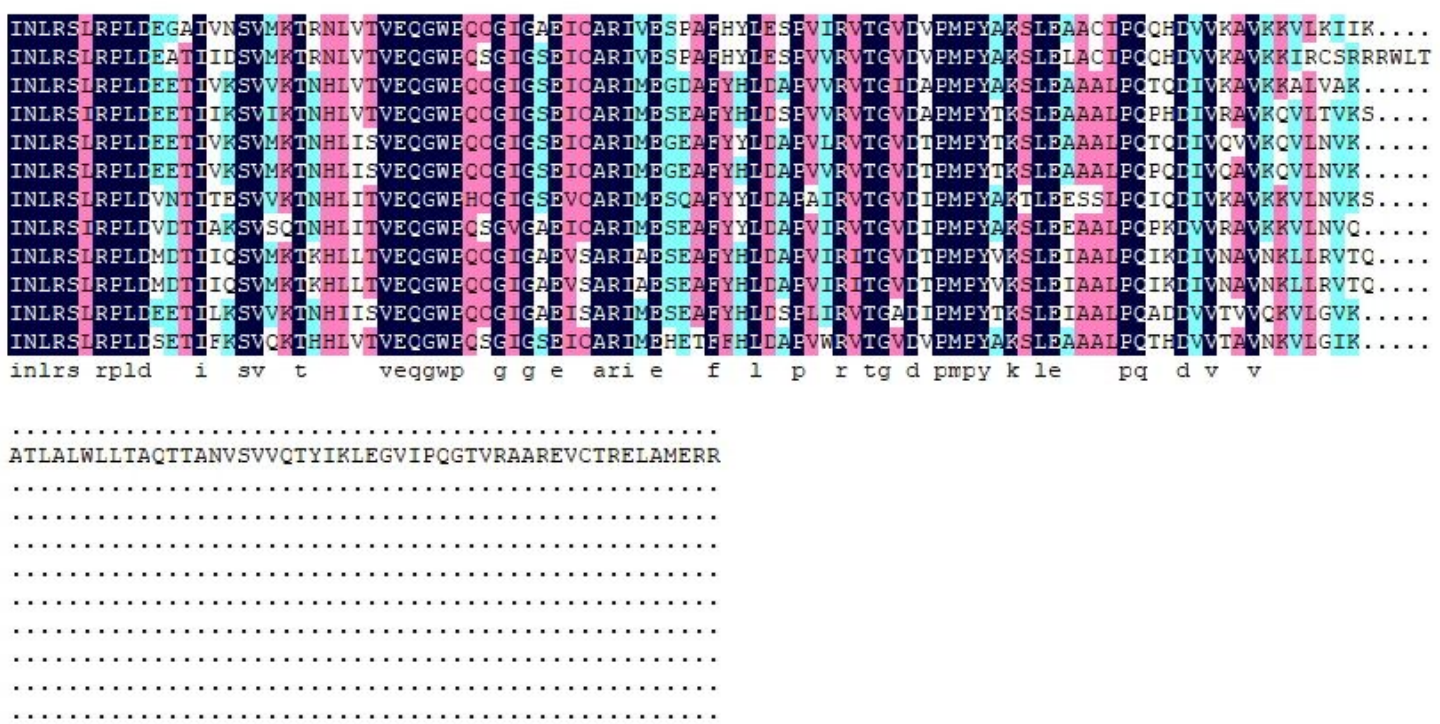

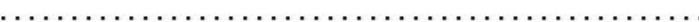

\section{Figure 2}

The alignment of amino acid sequences of PDHE1 from different species. 


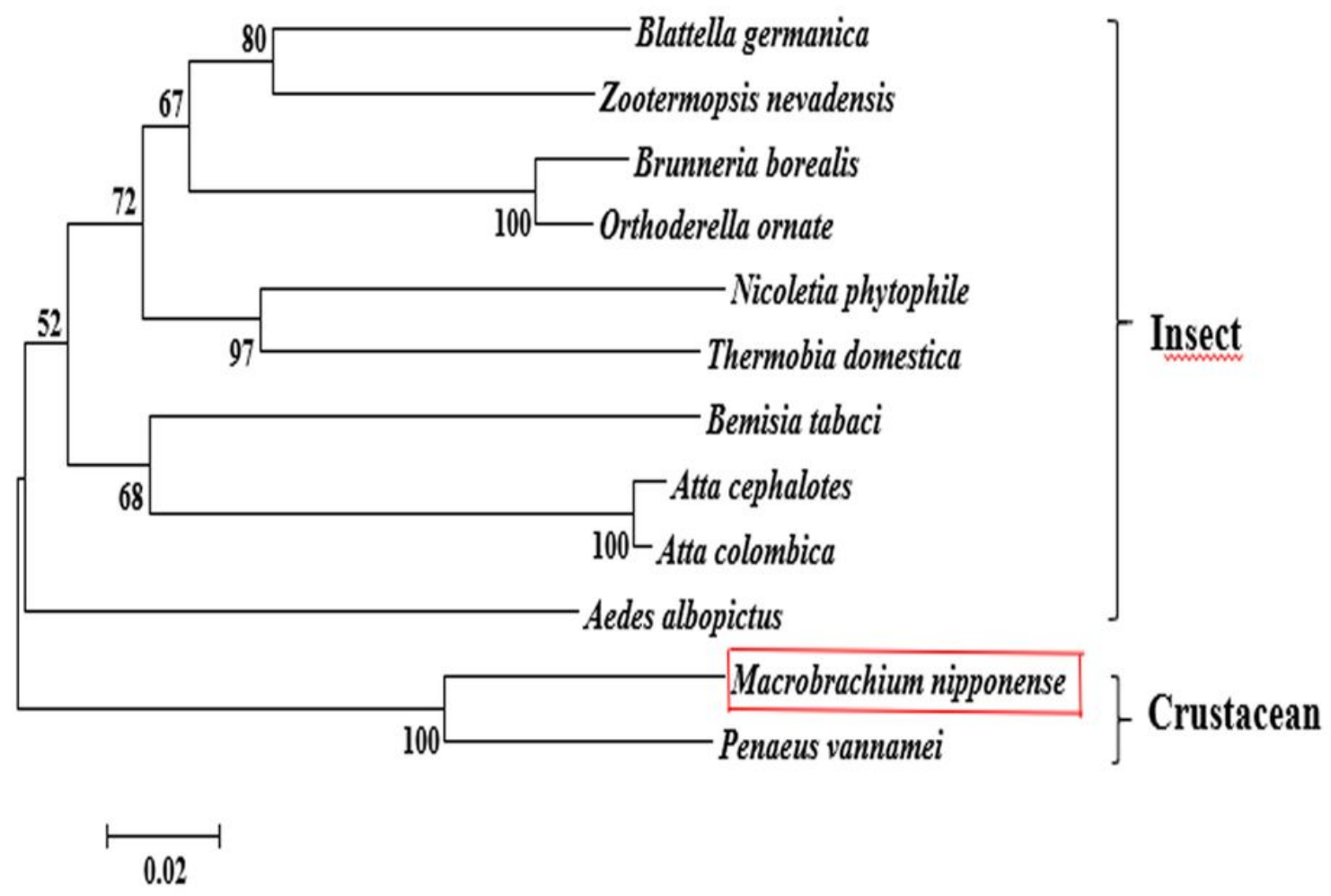

Figure 3

The phylogenetic tree of PDHE1 from different organisms based on amino acid sequence comparisons. Species names and types of PDHE1 are listed on the right of the tree. Red rectangles indicated M. nipponense. 

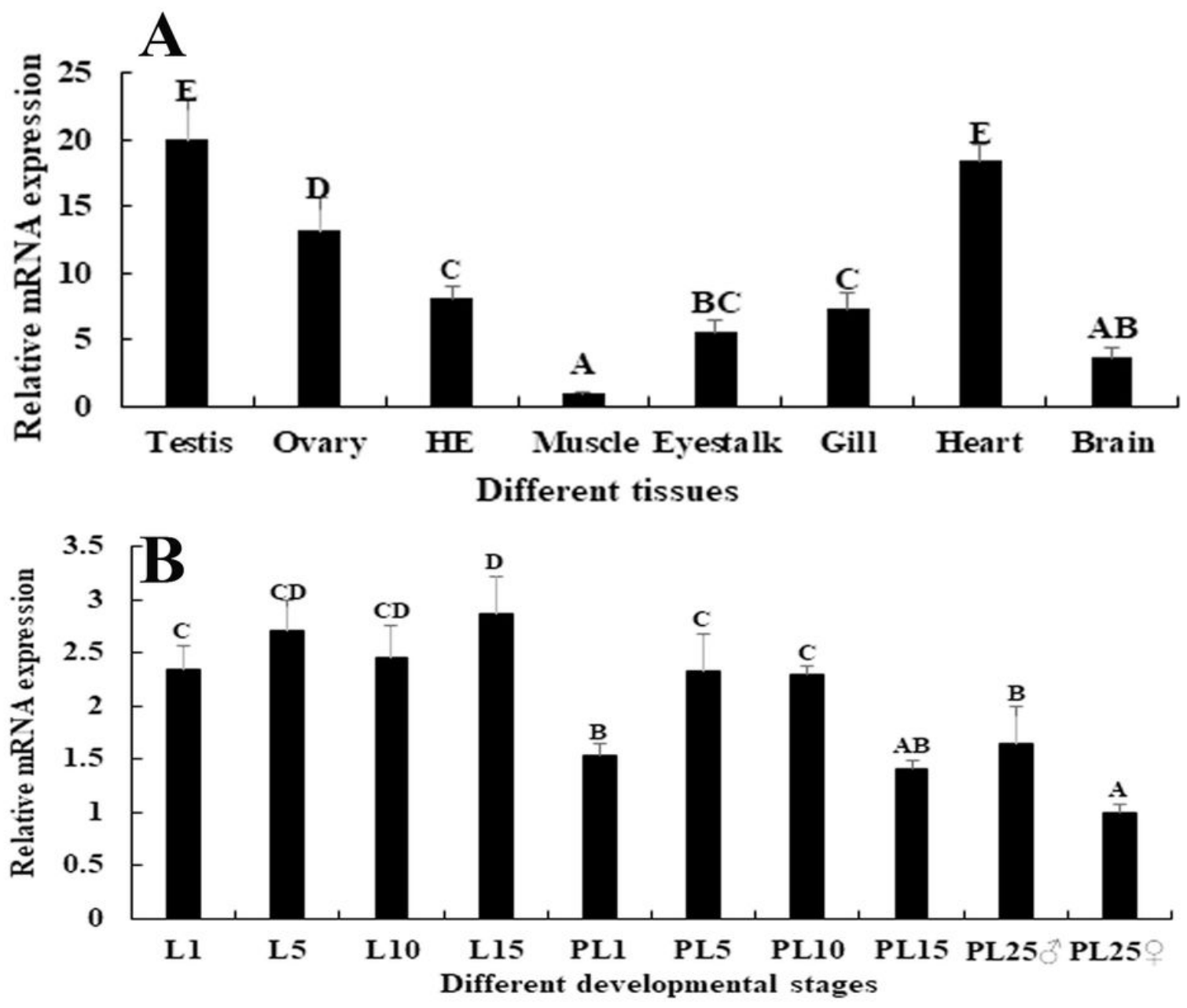

Figure 4

A: Expression characterization of Mn-PDHE1 in different tissues. Fig. 4-B: Expression characterization of Mn-PDHE1 in different developmental stages. The amount of Mn- PDHE1 mRNA was normalized to the EIF transcript level. Data are shown as mean +SD (standard deviation) of tissues from three separate individuals. Capital letters indicate expression difference between different samples. 


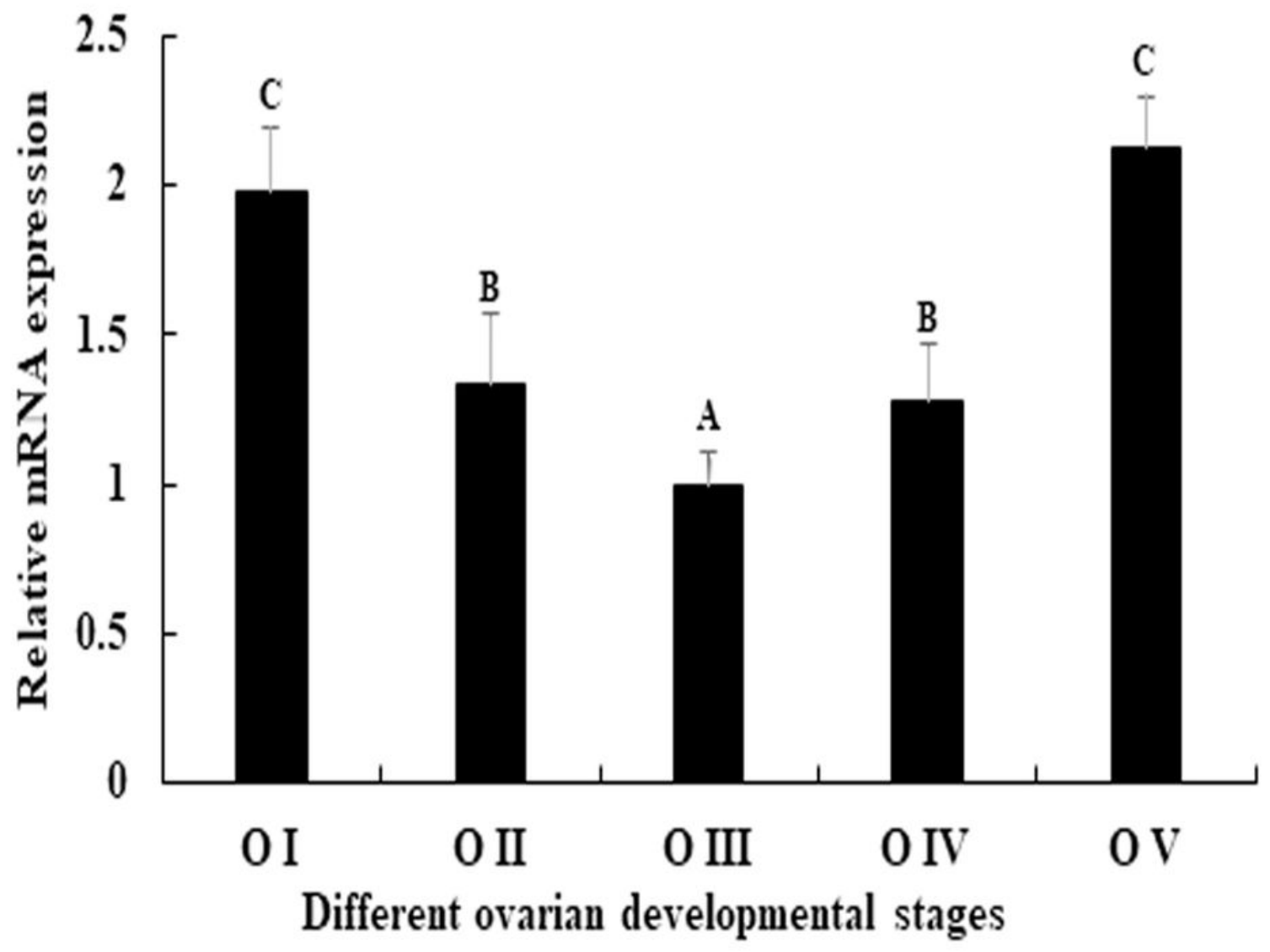

Figure 5

Expression characterization of Mn- PDHE1 in different reproductive cycles of ovary. The amount of MnPDHE1 mRNA was normalized to the EIF transcript level. Data are shown as mean +SD (standard deviation) of tissues from three separate individuals. Capital letters indicate expression difference between different samples. 
A

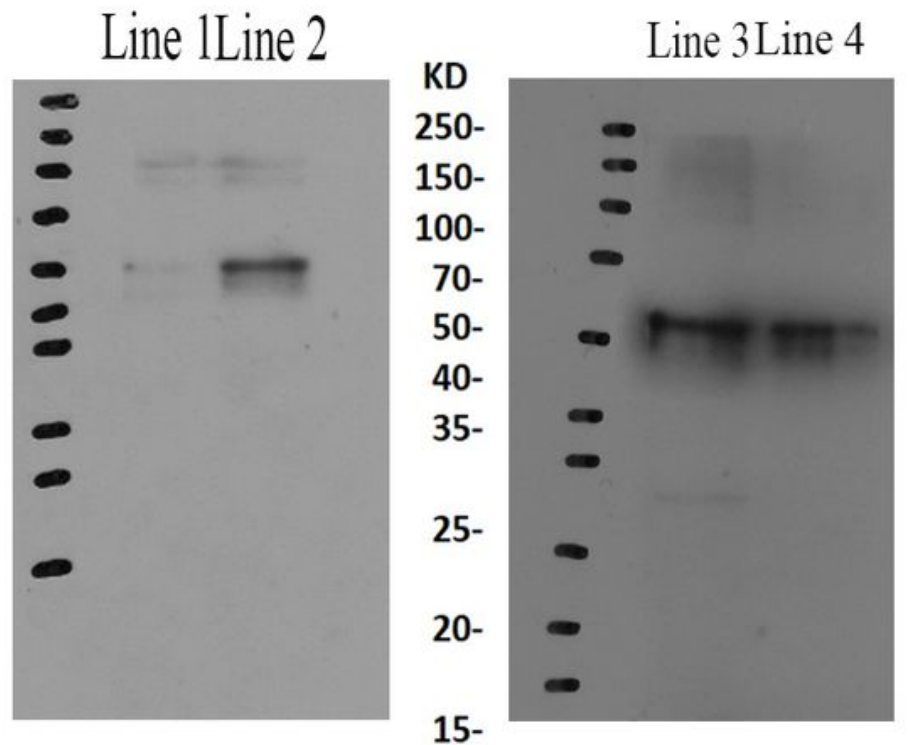

B

Line 1 Line 2 Line 3 Line 4 Line 5

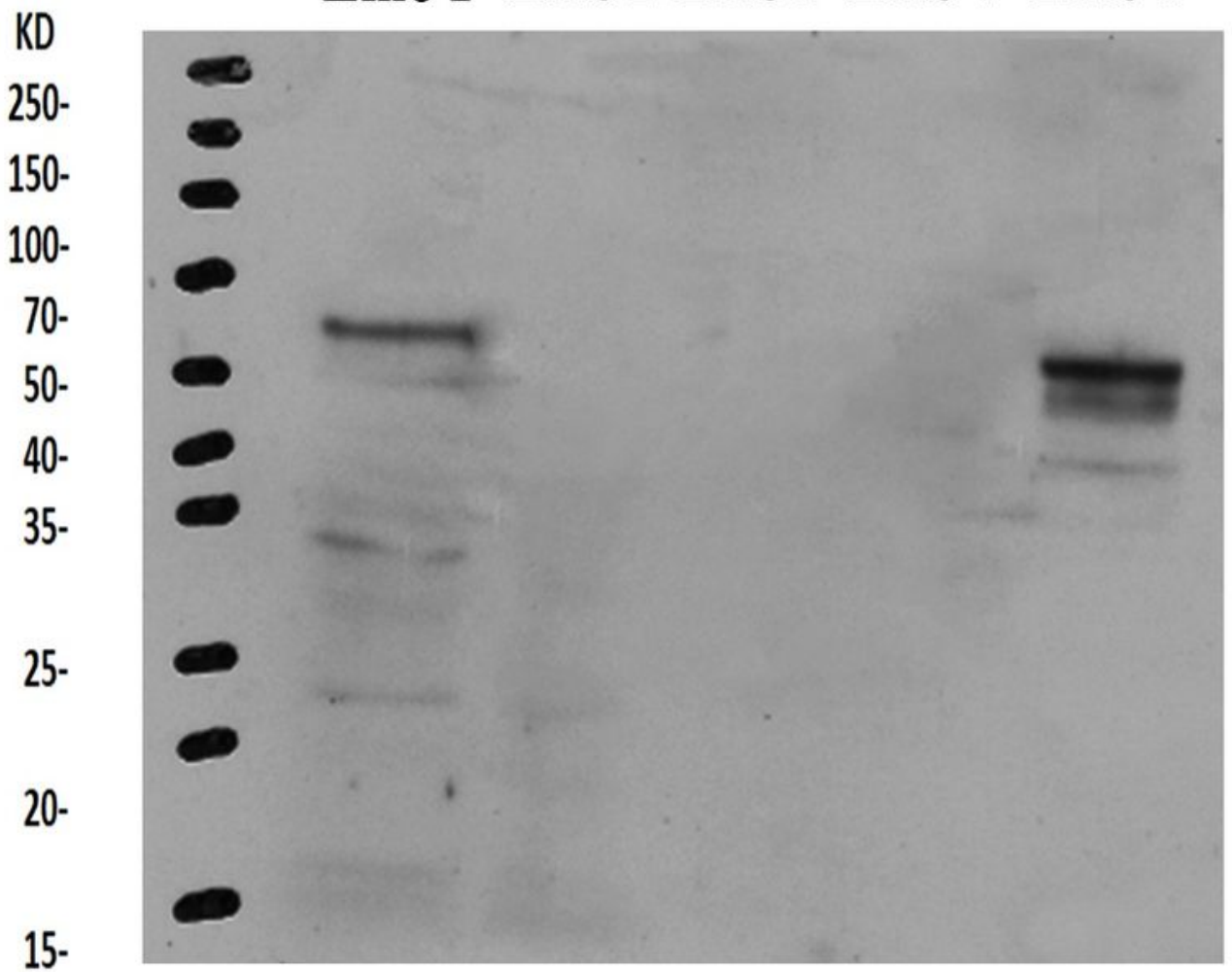

\section{Figure 6}

A: Western-blot analysis of Mn-PDHE1 in the testis and androgenic gland from the reproductive season and non-reproductive season. Line 1: Testis from the non-reproductive season; Line 2: Testis from the reproductive season; Line 3: Androgenic gland from reproductive season; Line 4: Androgenic gland from the non-reproductive season. Fig. 6-B: Western-blot analysis of Mn-PDHE1 in the different ovarian

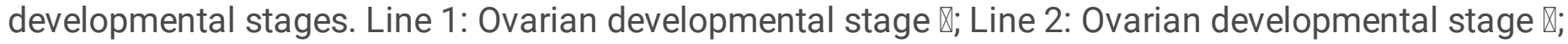




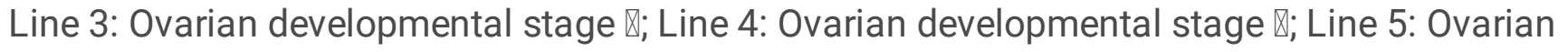
developmental stage $\mathrm{V}$. B-action was used as reference gene.
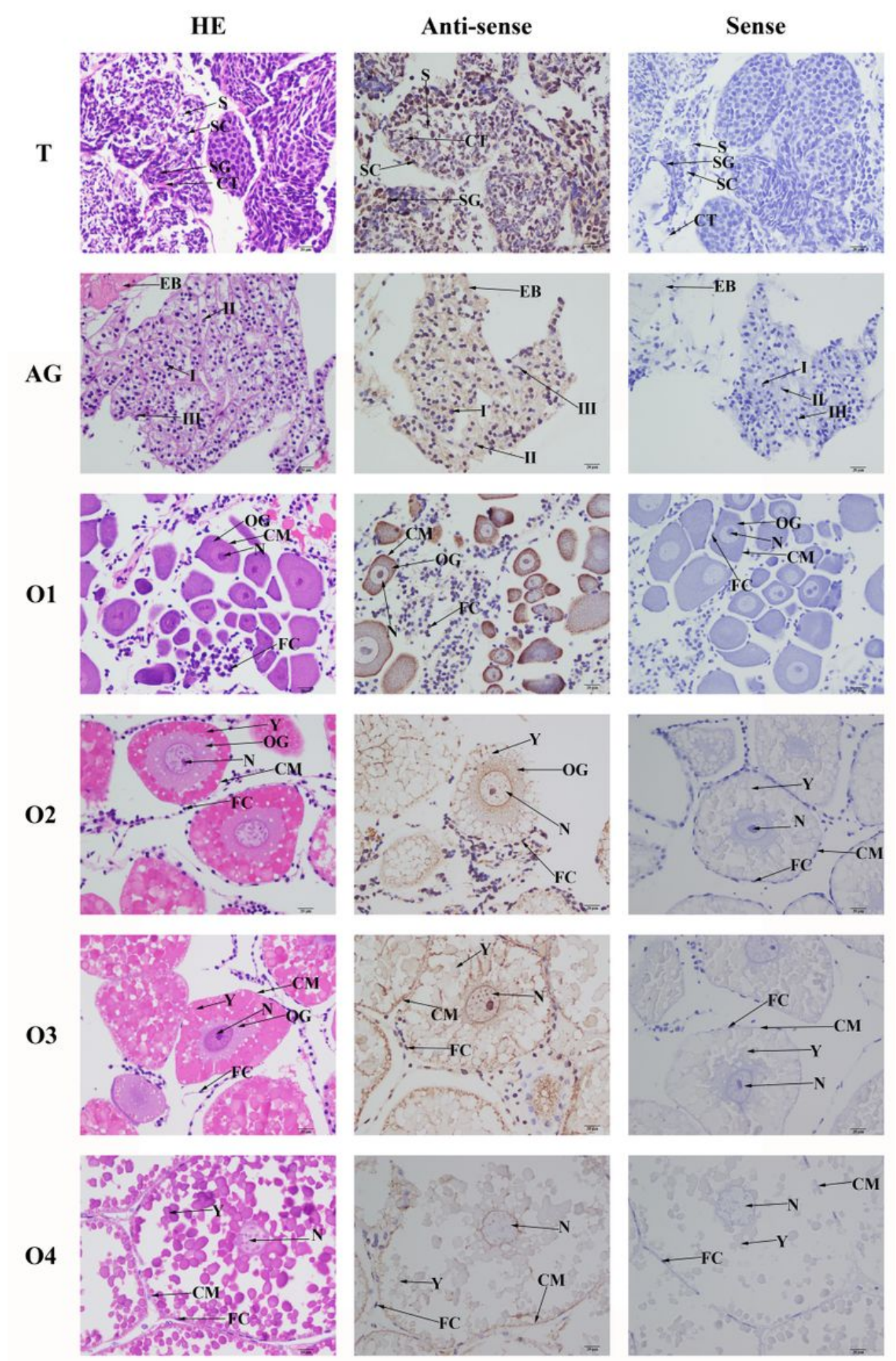

Figure 7

Location of PDHE1 gene was detected in testis, androgenic gland and ovary of M. nipponense by using in situ hybridization. ST: seminiferous tubule; SG: Spermatogonia; SC: spermatocyte; S: sperm; CT: collecting tissue; E: wall epithelium; EM: eosinophilic matrix; VD: vas deferens; EB: ejaculatory bulb; OG: 
oogonium; OC: oocyte; CM: cytoplasmic membrane; N: nucleus; Y: yolk granule; FC: follicle membrane. Scale bars $=20 \mu \mathrm{m}$.
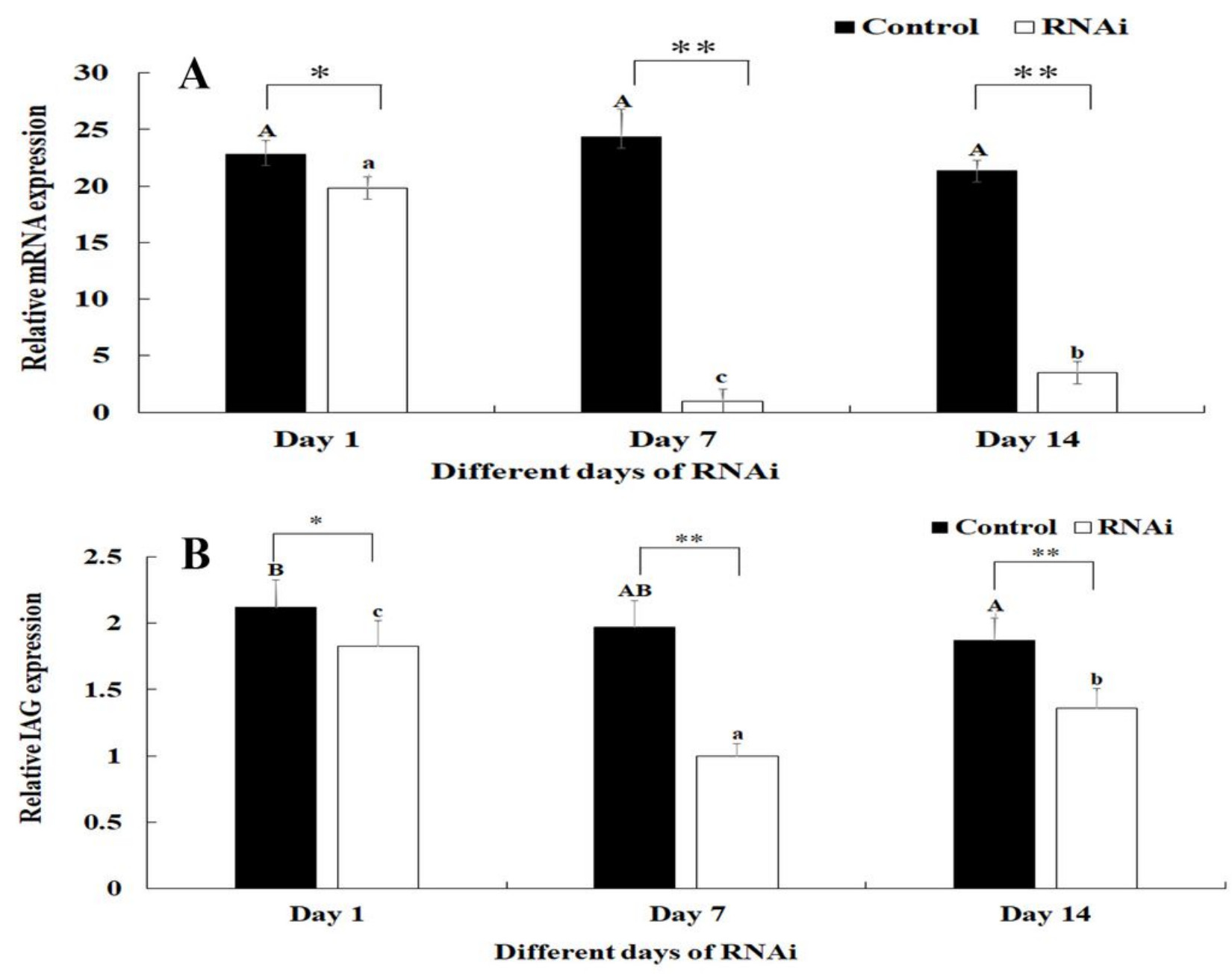

Figure 8

A: Expression characterization of Mn- PDHE1 at different days after Mn- PDHE1 dsRNA injection. Fig. 8-B: Expression characterization of Mn- IAG at different days after Mn-PDHE1 dsRNA injection. The amount of Mn- PDHE1 and Mn- IAG mRNA was normalized to the EIF transcript level. Data are shown as mean +SD (standard deviation) of tissues from three separate individuals. Capital letters indicated expression difference between different days after vehicle injection in control group. Lowercase indicated expression difference between different days after Mn- PDHE1 dsRNA injection in RNAi group. * $(P<0.05)$ and ** $(P<$ 0.01 ) indicates significant expression difference between the RNAi group and control group at the sample day. 


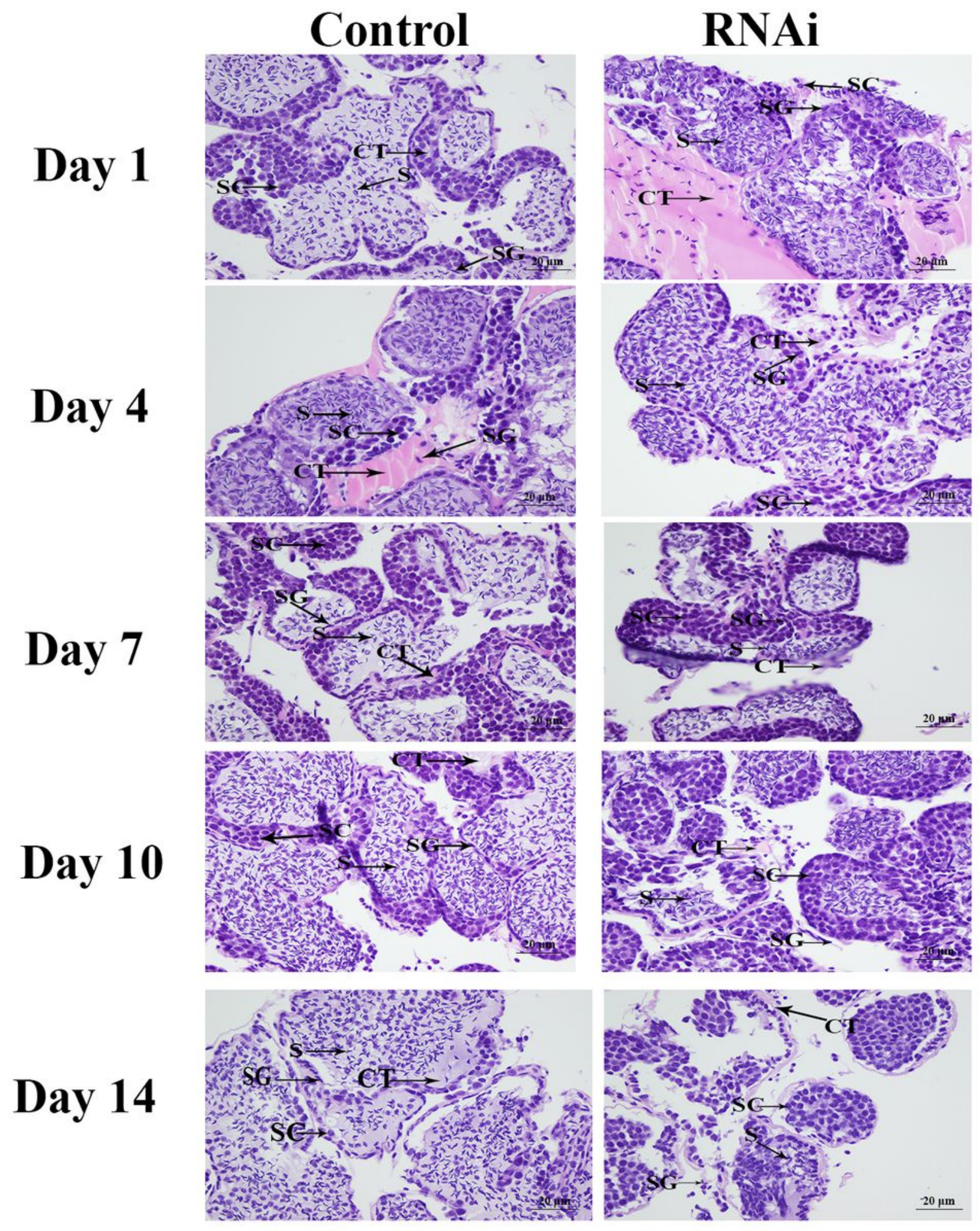

Figure 9

The histological observations of testis between RNAi and control group. SG: Spermatogonia; SC: spermatocyte; S: sperm; CT: collecting tissue. Scale bars $=20 \mu \mathrm{m}$. 


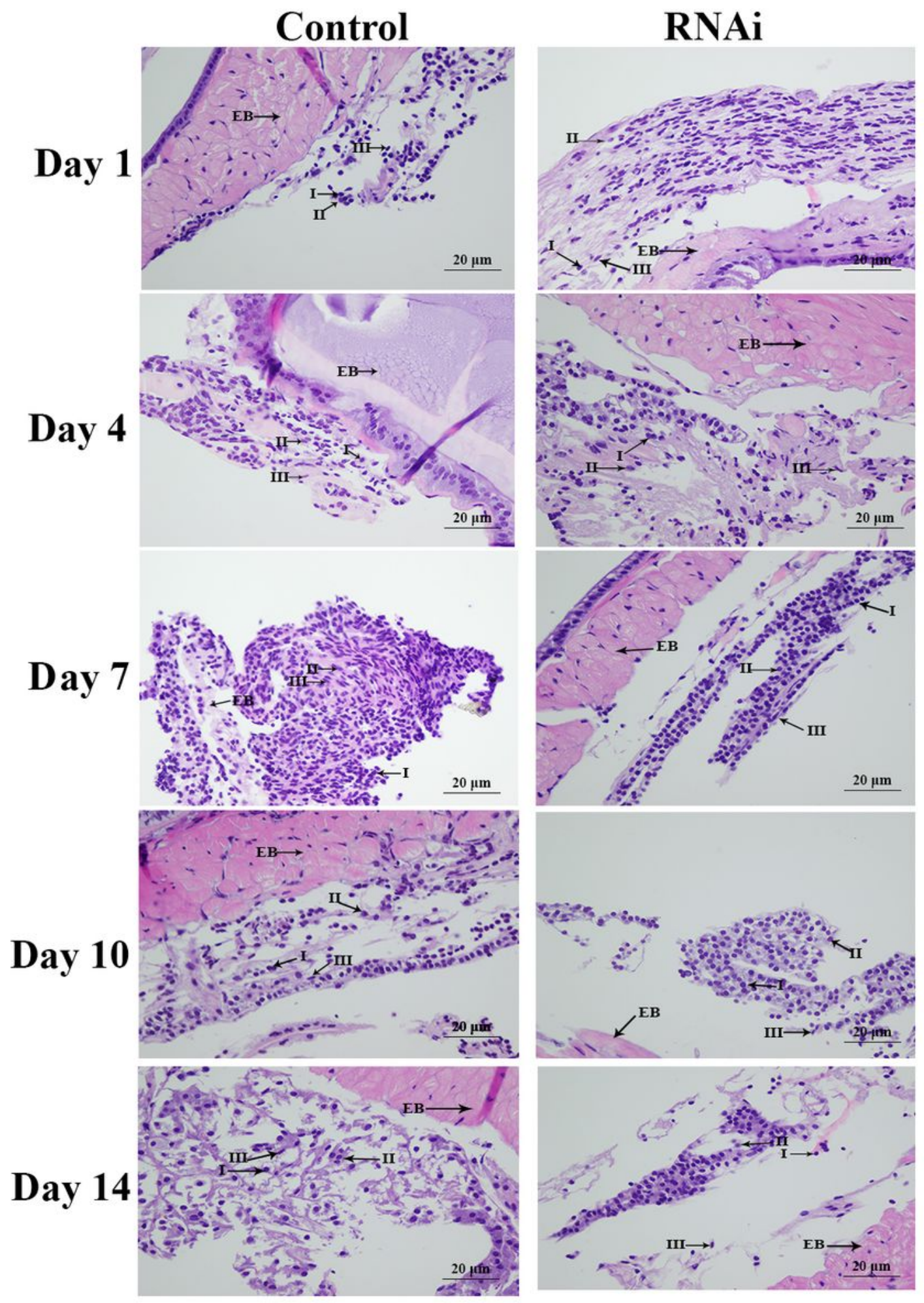

Figure 10

The histological observations of testis between RNAi and control group. EB: ejaculatory bulb; 『: Cell stage $\bigotimes$ of androgenic gland; $\Downarrow:$ Cell stage $\Downarrow$ of androgenic gland; $\Downarrow:$ Cell stage $\otimes$ of androgenic gland. Scale bars $=20 \mu \mathrm{m}$.

\section{Supplementary Files}


This is a list of supplementary files associated with this preprint. Click to download.

- Figure6Supplementaryimage.jpg

- Figure61Supplementaryimage.jpg 